$$
\text { CONF-96/141--7 }
$$

\title{
EFFECT OF PRESSURE ON THE MAGNETIC PHASE DIAGRAM OF THE ANTIFERROMAGNETIC SPIN-DENSITY-WAVE ALLOY Cr-1.6\% Si
}

\author{
J. A. Fernandez-Baca, \\ Oak Ridge National Laboratory, Oak Ridge, TN 37831-6393; \\ E. Fawcett, \\ University of Toronto, Toronto, Canada MSS 1A7, \\ H. L. Alberts, \\ Rand Afrikaans University, Johannesburg 2006, South Africa; \\ V. Yu Galkin, \\ Federal University of Parana, Curitiba, Brazil \\ and \\ Y. Endoh, \\ Tohoku University, Sendai 980-77, Japan

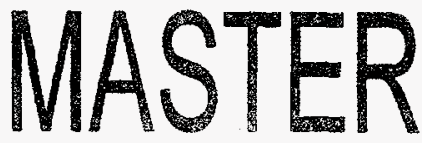

\begin{abstract}
A neutron diffraction experiment has been perionined to study the effects of high pressure on the magnetic phase diagram of a single crystal specimen of $\mathrm{Cr}-1.6 \% \mathrm{Si}$. At applied pressures up to $0.49 \mathrm{GPa}$ there are two clear transitions. At $\mathrm{T}_{\mathrm{NC}}$ there is a transition from a paramagnetic $(\mathrm{P})$ to a commensurate spin density wave (CSDW), while at lower temperatures there is a transition to a mixed state where the CSDW seems to coexist with an incommensurate SDW (ISDW) state. $\mathrm{T}_{\mathrm{NC}}$ decreases with the application of pressure while the transition temperature $\mathrm{T}_{\mathrm{CI}}$ to the mixed C-I state seems to be insensitive to pressure. At $0.69 \mathrm{GPa}$ the ISDW state disappears while the P-C transition changes drastically.

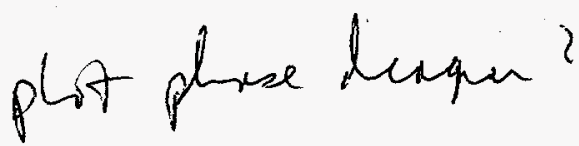

DISTRIBUTION OF THLS DOCUMENT IS UNLUMTEO

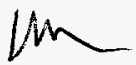

"The submitted manuscript has been authored by a contractor of the U.S. Government under contract No. DE-ACO5. 960R22464. Accordingly. the U.S. Government retains a non-exclusive, royalty-free license to publish or reproduce the published form of the contribution, or allow others to do so, for U.S. Government purposes."
\end{abstract}




\section{DISCLAIMER}

This report was prepared as an account of work sponsored by an agency of the United States Government. Neither the United States Government nor any agency thereof, nor any of their employees, makes any warranty, express or implied, or assumes any legal liability or responsibility for the accuracy, completeness, or usefulness of any information, apparatus, product, or process disclosed, or represents that its use would not infringe privately owned rights. Reference herein to any specific commercial product, process, or service by trade name, trademark, manufacturer, or otherwise does not necessarily constitute or imply its endorsement, recommendation, or favoring by the United States Government or any agency thereof. The views and opinions of authors expressed herein do not necessarily state or reflect those of the United States Government or any agency thereof. 


\section{DISCLAIMER}

Portions of this document may be illegible in electronic image products. Images are produced from the best available original document. 


\section{INTRODUCTION}

The spin density wave (SDW) antiferromagnetism in $\mathrm{Cr}$ is very sensitive to alloying.[1] This is explained by the changes in the Fermi-surface nesting that occur as different electron concentrations are introduced when atoms of different valence are alloyed with $\mathrm{Cr}$. The addition of only about $1 \%$ of $\mathrm{Si}$, for example, is needed to change the incommensurate spin density wave (ISDW) of pure $\mathrm{Cr}$ to a commensurate spin density wave (CSDW) state.[2] This transition from the paramagnetic to the CSDW is of strongly first-order character, as opposed to the weakly first-order iransition of the paramagnetic to the ISDW in pure Cr. [1] Another way to introduce significant changes in the SDW of the Cr-alloys is by the application of high pressure, indicating that the magnetoelastic effects in these systems are large.[1] The pressure dependence of the SDW phases in $\mathrm{Cr}$ Si systems was first studied Endoh et al. [3], who showed that the pressure-temperature phase diagram of $\mathrm{Cr}-1.4 \% \mathrm{Si}$ is rather complex. They found that the transition temperature to the CSDW phase in this system decreased rapidly with pressure, while at low pressures the transition temperature to a state in which the CSDW and ISDW regions coexist was almost independent of pressure. Above about $0.2 \mathrm{GPa}$ another mixed phase region appeared and the CSDW phase disappeared eventually at about 0.3 GPa. In order to have a better insight on the magnetoelastic effects of the $\mathrm{Cr}$-Si we have performed new neutron diffraction measurements on a single crystal of $\mathrm{Cr}-1.6 \% \mathrm{Si}$ under pressures up to $0.69 \mathrm{GPa}$. In this paper we report the preliminary results of these measurements.

\section{EXPERIMENTAL}

The sample utilized in this experiment was a single crystal of $\mathrm{Cr}-1.6 \% \mathrm{Si}$ grown by the floating zone technique using RF heating. This crystal cut from the same single crystal as the sample used in the measurements of Lourens and Alberts[4] and Anderson et al.[5]. The sample was cut down 
$\mathrm{T} \approx 100 \mathrm{~K}$. The first of these corresponds to the transition from the paramagnetic (P) to the CSDW state. We call this the P-C transition and its associated transition temperature $\mathrm{T}_{\mathrm{NC}}$. $\mathrm{T}_{\mathrm{NC}}$ decreases with the application of pressure from $T_{\mathrm{NC}} \approx 250 \mathrm{~K}$ at $\mathrm{P}=0$ to $\mathrm{T}_{\mathrm{NC}} \approx 180 \mathrm{~K}$ at $\mathrm{P}=0.49 \mathrm{GPa}$. The origin of the "rounded edge" at about $\mathrm{T} \approx 100 \mathrm{~K}$ is possibly related to concentration inhomogeneities in the sample. Despite these inhomogeneities the persistence of the $\left(\begin{array}{ll}1 & 0\end{array}\right)$ peak all the way down to the lowest temperatures indicates that most of the sample remains in the CSDWphase at $T=12 \mathrm{~K}$. The temperature dependence of the $\left(\begin{array}{lll}1 & 0 & 0\end{array}\right)$ peak at $P=$ $0.69 \mathrm{GPa}$ is very different to that at the lower pressures. The clear discontinuity of the $\mathrm{P}-\mathrm{C}$ transition disappears while the peak intensity increases continuously from about $\mathrm{T} \approx 150 \mathrm{~K}$ to $80 \mathrm{~K}$. Below $\mathrm{T} \approx 80 \mathrm{~K}$ there is another transition to a state that persists down to $\mathrm{T}=12 \mathrm{~K}$. This behavior might indicate that the P-C transition has acquired a second-order character or that , if it maintains its first-order character, it has shifted down to $\mathrm{T}_{\mathrm{N}}=80 \mathrm{~K}$. If this is the case the additional intensity between 80 and $150 \mathrm{~K}$ would be related to inhomogeneities in the sample concentration, this will be discussed in the following section.

Figure 2 shows that the main features of the ISDW state are very similar for $\mathrm{P}=0,0.27$ and $0.49 \mathrm{GPa}$. There is a relatively sharp transition at $\mathrm{T}_{\mathrm{CI}} \approx$ $100 \mathrm{~K}$ accompanied by a "tail" of intensity above this temperature. This tail is due, presumably, to inhomogeneities in the Si concentration in our sample. The relatively sudden increase in the ISDW below $T_{C I} \approx 100 \mathrm{~K}$ indicates a transition from the CSDW phase to a ISDW state (C-I transition). Several authors have reported that this transition has a first-order character, and the fact that we do not observe a sharp feature at this temperature may be attributed to inhomogeneities in the sample that have the effect to round this transition. In this pressure range $(P \leq 0.49 \mathrm{GPa}) \mathrm{T}_{\mathrm{CI}}$ is insensitive to changes in the applied pressure. From figures 1 and 2 it can be observed that below $\mathrm{T}_{\mathrm{CI}} \approx 100 \mathrm{~K}$ both the CSDW and ISDW states seem 
to coexist as suggested by Endoh et al.[3]. At $\mathrm{P}=0.69 \mathrm{Gpa}$ (see the last panel of Fig. 2) the ISDW disappears and the remaining weak intensity at low temperatures is perhaps the effect of inhomogeneities.

\section{DISCUSSION}

The observed pressure dependence in the $\mathrm{Cr}-1.6 \% \mathrm{Si}$ is very different to that observed in Cr-1.4\% [3] confirming that the behavior of the SDW in the $\mathrm{Cr}-\mathrm{Si}$ system is very sensitive to small changes of the impurity concentration. The observed magnetic behaviors of the CSDW and ISDW in $\mathrm{Cr}-1.6 \%$ between 0 and $0.49 \mathrm{GPa}$ are qualitatively similar. There is a firstorder $\mathrm{P}-\mathrm{C}$ transition at $\mathrm{T}_{\mathrm{NC}}$, where $\mathrm{T}_{\mathrm{NC}}$ decreases with the application of pressure from $250 \mathrm{~K}$ (at $\mathrm{P}=0$ ) to $180 \mathrm{~K}$ (at $\mathrm{P}=0.49 \mathrm{GPa}$ ). Below $\mathrm{T}_{\mathrm{NC}}$ the intensity of the CSDW peak increases monotonically until about $\mathrm{T} \approx 100 \mathrm{~K}$ where the intensity decreases in a "smooth" fashion (this is the "rounded edge" mentioned in the previous section). This intensity decrease indicates that part of the sample is undergoing a CSDW to ISDW transition, the fact that this feature is smooth is likely to be originated by small concentration inhomogeneities. In typical crystals of this size these inhomogeneities are not unusual. The magnetic phase diagram of the $\mathrm{Cr}_{1-\mathrm{x}} \mathrm{Si}_{\mathbf{x}}$ system (see figure 17 of Ref . [1]) indicates that in the concentration range in the vicinity of $1.6 \% \mathrm{Si} \mathrm{T}_{\mathrm{CI}}$ is very sensitive to concentration inhomogenities. Above $\mathrm{T}=100 \mathrm{~K}$ only very weak scattering is observed at the ISDW satellite positions (these are the weak tails of intensity above $\mathrm{T}=100 \mathrm{~K}$ shown in Fig. 2), and at the same temperature where the decrease of the CSDW peak intensity ends (about $\mathrm{T}=100 \mathrm{~K}$ ) the ISDW starts increasing rather sharply and remains constant all the way to the lowest temperatures. This transition is likely to be of first order but the sharp features expected for this transition could have been smeared slightly by the sample inhomogeneities. In any case it is apparent that below $T_{C I} \approx 100 \mathrm{~K}$ there is a coexistence of the CSDW and ISDW phases as suggested by Endoh et al 
[3]. Recently Galkin et al [8 ] have pointed out that mixed I-C states have also been observed in other $\mathrm{Cr}$ binary alloys and they suggest that this effect is related to the strongly first-order strain at the I-C phase transition in these systems, if this strain is anisotropic it would lead to a mixed state that minimizes the elastic energy in a manner analogous to the multidomain state of a ferromagnet that minimizes the magnetostatic energy. Anderson et al [5] also suggested that such a mixed I-C state might occur for $\mathrm{Cr}-1.2 \% \mathrm{Si}$, although this was not directly observed in their thermal expansion and sound velocity measurements for $\mathrm{Cr}-1.6 \% \mathrm{Si}$ or in those of Lourens and Alberts [4].

At $\mathrm{P}=0.69 \mathrm{Ga}$ the temperature dependence of the CSDW and ISDW intensities is very different than at the lower pressures. The sharp feature indicative of the P-C transition disappears while there is an indication of a continuous transition that starts at about $150 \mathrm{~K}$. A second and sharper transition occurs at about $\mathrm{T} \approx 80 \mathrm{~K}$. This may be an indication that the $\mathrm{P}-\mathrm{C}$ transition has become of second order character and that it occurs at $\mathrm{T}_{\mathrm{NC}} \approx 150 \mathrm{~K}$. If this is the case it is not clear what is the nature of the lower temperature transition. Alternatively, it is conceivable that the P-C transition has been moved to $\mathrm{T}_{\mathrm{NC}} \approx 80 \mathrm{~K}$ and it remains first-order and that the scattering between $80 \mathrm{~K}$ and $150 \mathrm{~K}$ is due to sample inhomogeneities. More experiments are needed to clarify this point. The ISDW at this pressure (see the last panel of fig. 2) is almost completely suppressed

The work at ORNL was supported by the U. S. Department of Energy under contract No. DE-AC05-96OR22464 with Lockheed Martin Energy Research Corp. The pressure cell equipment used in this experiment was provided by the Japanese government through the US-Japan Cooperative Program on Neutron Scattering. The authors would like to acknowledge valuable discussions with $\mathrm{J}$. W. Cable and $\mathrm{Y}$. Tsunoda. 
REFERENCES

[1] E. Fawcett et al., Rev. Mod. Phys 66, 25 (1994).

[2] J. W. Cable, J. Magn. Mag. Mater. 5, 112 (1977).

[3] Y. Endoh, J. Mizuki and Y. Ishikawa, J. Phy. Soc. Jpn. 51, 2826 (1982).

[4] J. A. J. Lourens and H. L. Alberts, Int. J. Mod. Phys. B (Singapore) vol. 7, no.1-3, pt.2, p.630 (1993).

[5] R. A. Anderson, H. L. Alberts and P. Smith, J. Phys. Condes. Matter 5, 1732 (1993)

[6] Fluorinert is a fluorocarbon manufactured by Sumitomo Chem. Co.

[7] A. Onodera , Y. Nakai, N. Kunitomi, O. A. Pringle, H. G. Smith, R. M. Nicklow, R. M. Moon, F. Amita, N. Yamamoto, S. Kawano, N. Achiwa, and Y. Endoh, Jpn. J. Appl. Phys. 26, 152 (1987).

[8] V. Yu. Galkin, Camargo, N. Ali, E. Fawcett, these proceedings. 


\section{FIGURE CAPTIONS}

Figure 1. Plots of the peak intensity of the (1.00) reflection vs. temperature for $P=0,0.27$, $0.49,0.69 \mathrm{GPa}$. This peak corresponds to the CSDW in this system. The solid circles correspond to measurements taken while the sample was warming slowly. The open circles correspond to measurements performed after the sample was at the set temperature and time was allowed to assure thermal equilibrium.

$$
\begin{aligned}
& \text { Deod theme correstel' } \\
& \text { eur bers } 3 \text { (top pal) }
\end{aligned}
$$

Figure 2. Plots of the peak intensity of the $(1+\delta 00)$ satellite reflection vs. temperature for $\mathrm{P}=0,0.27,0.49,0.69 \mathrm{GPa}$. This peak corresponds to the ISDW in this system. The solid and open circles have the same meaning as in figure 1. 


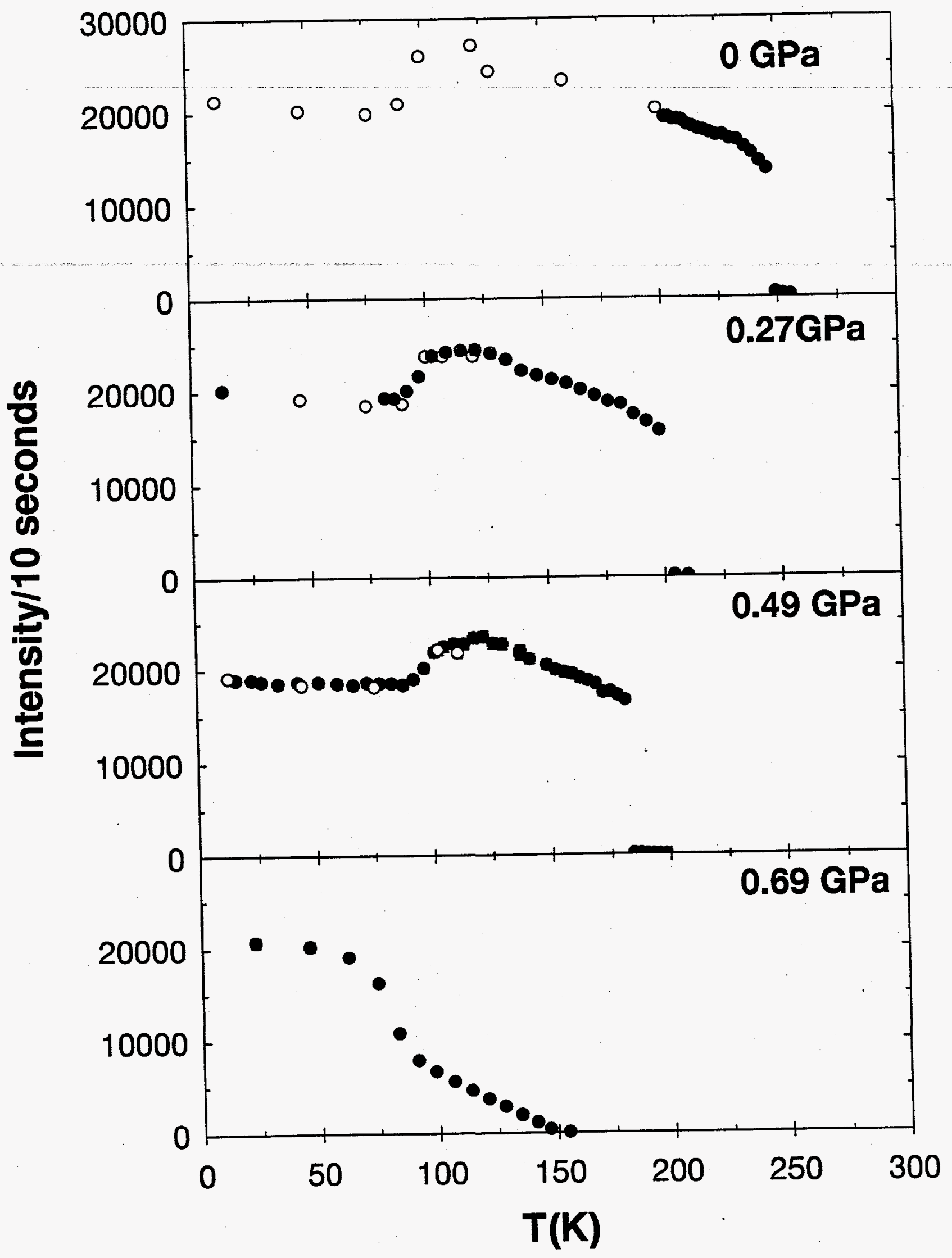

AE-11

J. A. Fernandez-Baca

Figure 1

MMM-96 


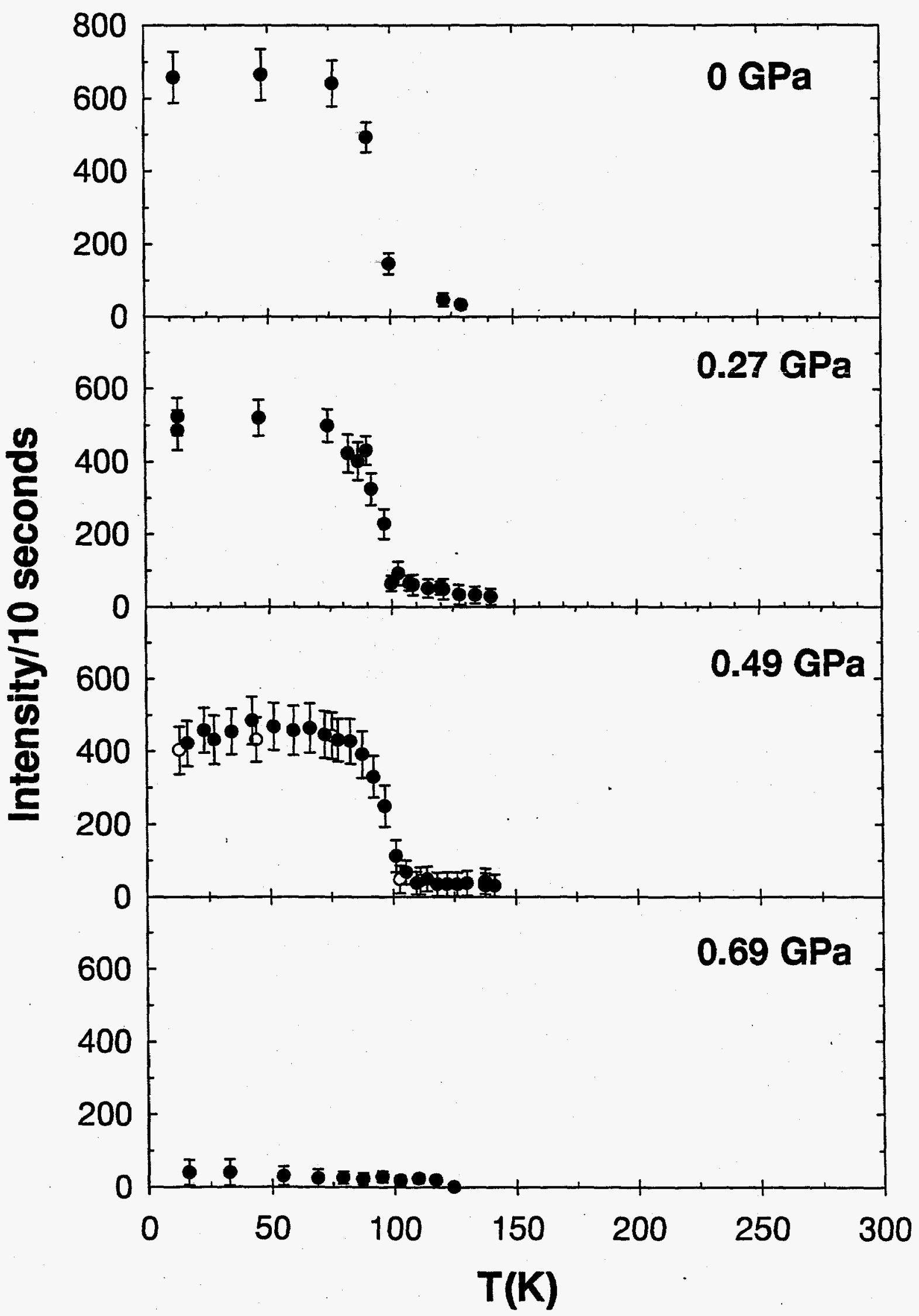

AE-11

Figure 2

J. A. Fernandez-Baca MMM-96 